\title{
Summary of session B3: analytic approximations, perturbation methods and their applications
}

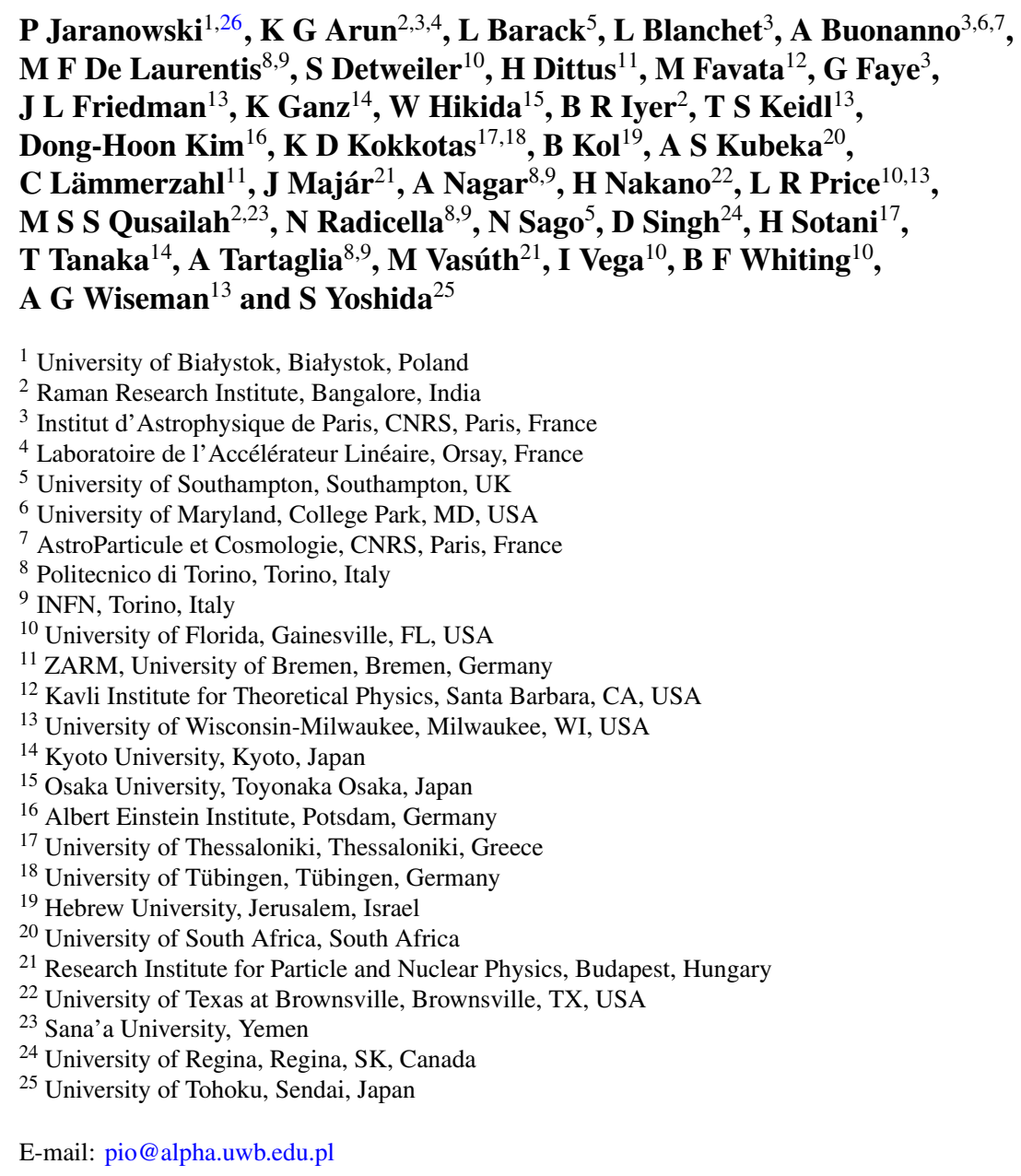

Received 30 October 2007, in final form 2 November 2007

Published 15 May 2008

Online at stacks.iop.org/CQG/25/114020

\section{Abstract}

The paper summarizes the parallel session B3 analytic approximations, perturbation methods and their applications of the GR18 conference. The talks

\footnotetext{
${ }^{26} \mathrm{~B} 3$ session's chairman.
} 
in the session reported notably recent advances in black hole perturbations and post-Newtonian approximations as applied to sources of gravitational waves.

PACS numbers: 04.25.Nx, 04.25.-g, 04.30.-w, 04.30.Db

\section{Introduction}

Analytic approximations and perturbation methods are of common use in different branches of general relativity theory. The most common methods are the post-Newtonian approximations, able to deal with the gravitational field of any system in the non-relativistic limit, the postMinkowskian approximations, which are appropriate for relativistic systems in the weak gravitational field regime, and the perturbation formalisms, which expand (at linear order in general) around some exact solution of the Einstein field equations. The B3 session analytic approximations, perturbation methods and their applications of GR18 conference was dominated by the issues more or less directly related to the problem of detecting gravitational waves by currently operating or planned to be built in the near future detectors. One of the most important targets for gravitational-wave detectors is coalescing binary systems made of compact objects of different kinds. These sources produce 'chirps' of gravitational radiation whose amplitude and frequency are increasing in time. For successful detection of the chirp signals and extraction of their astrophysically important parameters it is crucial to theoretically predict gravitational waveforms with sufficient accuracy.

One of the important sources for the future space-based LISA detector is extrememass-ratio binaries consisting of a small compact body (of stellar mass) orbiting around a supermassive black hole. The need for accurate modeling of the orbital dynamics of such binaries motivates some recent work on the problem of calculating the gravitational self-force experienced by a point particle moving in the background spacetime of a more massive body. Several talks in the session reported the progress on different aspects of the gravitational self-force computations. These were talks by Detweiler, Favata, Friedman, Hikida, Sago and Whiting.

For comparable-mass binaries, computations based on the post-Newtonian approximation of general relativity are useful for constructing gravitational-wave templates for data-analysis purposes. The post-Newtonian approximation describes with great accuracy the inspiral phase of these systems. The analytic post-Newtonian results are currently matched to recent numerical calculations of the merger and ring-down phases of black hole binaries [1,2]. In the contributed talks (by Blanchet, Iyer, and Vasúth) recent results on incorporating the spin-orbit effects (also within the accuracy beyond the leading-order effects) as well as the generalization to eccentric orbits (most of the explicit analytic results concern circular orbits) were presented.

Recently, a new approach to the perturbative solution of the problem of motion and radiation in general relativity was developed. This is the approach pioneered by Goldberger and Rothstein [3] in which effective field theory methods were used to describe the dynamics of nonrelativistic extended objects coupled to gravity. Kol discussed this approach as well as some of its applications. A Tartaglia presented a new semi-analytic method for computing the emission of gravitational waves by a compact object moving in the background of a more massive body.

Several other topics were tackled in the contributed talks. Lämmerzahl discussed the influence of the cosmic expansion on the physics in gravitationally bound systems. Singh presented an analytic perturbation approach for classical spinning particle dynamics, based on the Mathisson-Papapetrou-Dixon equations of motion. Sotani studied gravitational radiation 
from collapsing magnetized dust. Kubeka remarked on the computation of the Ricci tensor for non-stationary axisymmetric spacetimes.

In the rest of this paper all talks contributed to the $\mathrm{B} 3$ session are sketched in more detail (in the order in which they were presented at the conference).

\section{Contributed talks}

\subsection{Self-force analysis in extreme-mass-ratio inspiral by Detweiler and Vega (reported by Detweiler)}

The motion of a small object of mass $m$ orbiting a supermassive black hole of mass $M$ deviates slightly from a geodesic and has an acceleration that scales as the ratio $m / M$ of the masses. This acceleration includes the dissipative effects of radiation reaction and is said to result from the gravitational self-force acting on $m$ [4]. As an alternative, the effects of the self-force may be described as geodesic motion in an appropriately regularized metric of the perturbed spacetime [5]. The LISA effort requires accurate gravitational wave templates for data analysis. For extreme-mass-ratio inspirals the templates should include both the dissipative and conservative effects of the self-force.

The talk described a novel, efficient method for simultaneously calculating both the gravitational self-force and its effect on the gravitational waveform. The authors replaced the usual singular point source with a distributed, abstract, analytically determined source. The resulting perturbation in the field from this special distributed source is guaranteed to be differentiable at the location of the particle and to provide the appropriate self-force effect on the motion of the particle. At the same time, the field from the distributed source is identically equal to the actual perturbed field in the wave zone. Thus, this abstract field simultaneously provides both the self-force acting on a point source and also the effect of the self-force on the waveform of the radiation.

\subsection{The adiabatic approximation and three-body effects in extreme-mass-ratio inspirals by Favata}

Extreme-mass-ratio inspirals (EMRIs) are an important class of LISA sources consisting of a compact object inspiralling into a supermassive black hole. The detection of these sources and the precision measurement of their parameters rely on the accurate modeling of their orbital dynamics. A precise description of the binary's orbit requires an evaluation of the compact object's self-force. The adiabatic approximation (more appropriately referred to as the radiative approximation $[6,7]$ ) consists of computing the time-averaged rates of change of the three conserved quantities of geodesic motion. Its use greatly simplifies the computation of the orbital evolution. However, the adiabatic approximation ignores corrections to the conservative dynamics proportional to the mass $m$ of the compact object. These 'postadiabatic' corrections will affect the binary's positional orbital elements, for example, by causing $O(m)$ corrections to the pericenter precession rate.

Using a toy model of an electric charge orbiting a central mass and perturbed by the electromagnetic self-force, Pound, Poisson and Nickel [8] have called into question the accuracy of the adiabatic approximation, especially for eccentric orbits. In order to estimate the size of the post-adiabatic phase errors in the gravitational case, the author presented an analytical computation, accurate to second-post-Newtonian order, of the small-eccentricity corrections to the gravitational-wave phase. These post-Newtonian, eccentricity corrections to the phase can be significant not only for EMRIs but for other binary sources as well. Using this phase expansion it was found that the post-adiabatic phase errors are sufficiently small 
that waveforms based on the adiabatic approximation can be used for EMRI detection, but not for precise parameter measurements.

The author also discussed the effect of a third mass orbiting the EMRI. The analysis models the system as a hierarchical triple using the equations of motion of Blaes, Lee and Socrates [9]. To have a measurable effect on the EMRI's waveform, the distant mass must be sufficiently close to the compact object that both the inner and outer binaries would be detected as EMRIs. Such 'double-EMRI' systems are rare.

\subsection{Extreme-mass-ratio binary inspiral in a radiation gauge by Friedman, Keidl, Dong-Hoon Kim, Messaritaki and Wiseman (reported by Friedman)}

Gravitational waves from the inspiral of a stellar-size black hole of mass $m$ to a supermassive black hole of mass $M$ can be accurately approximated by a point particle moving in a Kerr background. To find the particle's orbit to first order in the mass ratio $m / M$, one must compute the self-force. The computation requires a renormalization, but the well-known MiSaTaQuWa prescription $[10,11]$ involves a harmonic gauge, a gauge that is not well suited to perturbations of the Kerr geometry. In a harmonic gauge, one solves ten coupled PDEs, instead of the single decoupled Teukolsky equation for the gauge-invariant components $\left(\psi_{0}\right.$ or $\left.\psi_{4}\right)$ of the perturbed Weyl tensor. In the talk progress was reported in finding the renormalized self-force from $\psi_{0}$ or $\psi_{4}$. Following earlier work by Chrzanowski and by Cohen and Kegeles, a radiation gauge was adopted to reconstruct the perturbed metric from the perturbed Weyl tensor. The Weyl tensor component is renormalized by subtracting a singular part obtained using a recent DetweilerWhiting version [12] of the singular part of the perturbed metric as a local solution to the perturbed Einstein equations. The authors' method relies on the fact that the corresponding renormalized $\psi_{0}$ is a sourcefree solution to the Teukolsky equation. One can then reconstruct a nonsingular renormalized metric in a radiation gauge, a gauge that exists only for vacuum perturbations. More details can be found in [13].

\subsection{Adiabatic evolution of three 'constants' of motion in Kerr spacetime by Hikida, Ganz, Nakano, Sago and Tanaka (reported by Hikida)}

General orbits of a particle of small mass around a Kerr black hole are characterized by three parameters: the energy, the angular momentum and the Carter constant. For energy and angular momentum, one can evaluate their change rates from the fluxes of the energy and the angular momentum at infinity and on the event horizon according to the balance argument. On the other hand, for the Carter constant, one cannot use the balance argument because the conserved current associated with it is not known. Recently, Mino proposed a new method of evaluating the averaged change rate of the Carter constant by using the radiative field. The authors developed a simplified scheme for practical evaluation of the evolution of the Carter constant based on the Mino's proposal. In the talk this scheme was described in some detail, and derivation of explicit analytic formulae for the change rates of the energy, the angular momentum and the Carter constant was presented. Also some numerical results for large eccentric orbits were shown. For more details see [14].

\subsection{Gravitational self-force on a particle orbiting a Schwarzschild black hole by Barack and Sago (reported by Sago)}

In the talk the calculation of the gravitational self-force acting on a pointlike particle moving around a Schwarzschild black hole was presented. The calculation was done in the Lorenz gauge. First, the Lorenz-gauge metric perturbation equations were solved directly using 
numerical evolution in the time domain. Then the back-reaction force from each of the multipole modes of the perturbation was computed. Finally, the mode sum scheme was applied to obtain the physical self-force. The temporal component of the self-force describes the rate of the loss of orbital energy. As a check of their scheme, the authors compared their result for this component with the total flux of gravitational-wave energy radiated to infinity and through the event horizon. The radial component of the self-force was also calculated. From their result for the self-force, the authors computed the correction to the orbital frequency due to the gravitational self-force, taking into account both the dissipative and the conservative effects. More details can be found in [15].

\subsection{Mobile quadrupole as a semianalytic method for gravitational-wave emission by Tartaglia, De Laurentis, Nagar and Radicella (reported by Tartaglia)}

The quadrupole formula is the simplest approximation for studying the gravitational-wave emission from a binary system. The formula gives its best performance for quasi-circular and quasi-stationary motion of the emitters. Whenever the motion is far from the quasi-circular approximation, other semi-analytic methods or numerical calculations of growing complexity must be used. In the talk a situation was studied where the gravitational wave is emitted by a concentrated object accelerating in the background field of a central mass. Provided one knows the background metric, the gravitational wave represents a first-order perturbation on it, so that the spacetime trajectory of each object of the pair is almost geodesic. Once the equations of a geodesic are written, the motion can be thought of as an instantaneous rotation around an (instantaneously at rest) curvature center for the space trajectory. In this condition the quadrupole formula is easily applicable at each different place along the geodesic, after calculating the curvature and the equivalent angular velocity as the ratio between the threespeed and the curvature radius. Everything is reduced to a problem of ordinary geometry. The energy emission rate and the waveforms obtained by this way must simply be converted from the local time to the time of a far away inertial observer. The approach was applied to the capture of a mass by a Kerr black hole. The method is much lighter (from the computational point of view) than numerical relativity, giving comparable results. For the research results relevant to the presented approach see [16-18].

\subsection{The non-radiated multipoles in the perturbed Kerr spacetime by Price and Whiting (reported by Whiting)}

For the self-force problem in general relativity, it has been shown that the perturbed metric produced by a finite-mass test, point-particle, has a singular part which exerts no influence on the particle, while the self-force which the particle experiences arises entirely due to a metric perturbation which is smooth at the location of the particle [12]. However, metric reconstruction from the perturbed Weyl tensor is unable to yield perturbations for the nonradiated multipoles in Petrov type II spacetimes, such as that surrounding the Kerr black hole [19]. In the talk a new form of the perturbed Einstein equations, developed by the authors on the basis of the Newman-Penrose formalism, was presented. With its assistance, progress toward filling the low multipole gap, which will contribute to the calculation of regularization parameters for the self-force problem, was discussed.

\subsection{Higher-order spin effects in the radiation field of compact binaries by Blanchet, Buonanno and Faye (reported by Blanchet)}

In the talk the investigation, motivated by the search for gravitational waves emitted by binary black holes, of the gravitational radiation field of compact objects with spins was discussed. 
The approach is based on the multipolar post-Newtonian wave generation formalism and on the formalism of point particles with spin (Papapetrou-Dixon-Bailey-Israel). The authors computed: (i) the spin-orbit coupling effect in the binary's equations of motion one postNewtonian (PN) order beyond the dominant effect (confirming a previous result by Tagoshi et al [20]), (ii) the spin-orbit coupling effects in the binary's mass and current quadrupole moments at the same order, (iii) the spin-orbit contributions in the gravitational-wave energy flux and (iv) the secular evolution of the binary's orbital phase up to 2.5PN order (for maximally rotating black holes). Previous results on the spin-orbit effect at the lowest order were computed in [21, 22]. Crucial ingredients for obtaining the next-order 2.5PN contribution in the orbital phase are the binary's energy and the spin precession equations. These results provide more accurate gravitational-wave templates to be used in the data analysis of rapidly rotating Kerr-type black-hole binaries with the ground-based interferometric detectors and the space-based detector LISA. Details of the presented results were published in [23, 24].

\subsection{The 3PN gravitational wave luminosity from inspiralling compact binaries in eccentric orbits by Arun, Blanchet, Iyer and Qusailah (reported by Iyer)}

Some details of the computation of the complete gravitational-wave luminosity of inspiralling compact binaries on quasi-elliptical orbits up to the third post-Newtonian (3PN) order using multipolar post-Minkowskian formalism were presented. There are two types of contributions to the gravitational-wave luminosity at 3PN order: the instantaneous-type terms, which depend on the dynamics of the binary only at the retarded instant, and the hereditary terms, which are sensitive to dynamics of the system in the entire past. The new inputs for the calculation of the $3 \mathrm{PN}$ instantaneous terms include the mass octupole and current quadrupole at 2PN for general orbits and the 3PN accurate mass quadrupole. Using the 3PN quasi-Keplerian representation of elliptical orbits obtained recently [25], the flux is averaged over the binary's orbit. The hereditary terms have a 'tail', 'tail of tail' and 'tail-squared' contributions which are computed using a semi-analytic procedure extending the earlier work of Blanchet and Schäfer at 1.5PN [26]. This semi-analytic extension uses the 1PN quasi-Keplerian parametrization of the binary and exploits the doubly periodic nature of the orbital motion. The final 3PN accurate energy flux averaged over the binary's orbit was presented in the modified harmonic (which contains no logarithmic terms) and the ADM coordinates. Also a gauge-invariant expression of the flux was provided in terms of the orbital frequency and the periastron precession constant. The results are consistent with those obtained by perturbation theory in the test particle limit to order $e_{t}^{2}$ (where $e_{t}$ is the so-called time eccentricity) and the 3PN circular orbit results. These results form the starting input for the construction of templates for inspiralling binaries in quasi-eccentric orbits, an astrophysically possible class of sources for both the ground-based and the space-based gravitational-wave interferometers. More detail can be found in [27].

\subsection{On the influence of the cosmic expansion on the physics in gravitationally bound systems by Lämmerzahl and Dittus (reported by Lämmerzahl)}

It is an old question whether the cosmological expansion influences the dynamics of gravitationally bound systems [27]. Though sometimes it has been claimed that the expansion will tie apart gravitationally bound systems, the majority of papers covering this issue derive no measurable influence. In the talk some additional arguments for the latter were given. It was shown that (i) the gravitational field created by an isolated body will feel only a tiny influence, (ii) the planetary orbits also are practically inert against the expansion and 
(iii) Doppler tracking of satellites in deep space is also only marginally influenced by the cosmic expansion.

\subsection{Spin evolution in binary systems by Vasúth and Majár (reported by Vasúth)}

Gravitational waves emitted by compact binary systems are characterized by different parameters of the binary. Among them the effects of rotation of the orbiting bodies appear at 1.5 post-Newtonian (PN) order both in the dynamical description and in the wave generation problem. In the talk the evolution of the individual spins of the bodies was discussed for compact binaries in circular and general eccentric orbits. For a $2 \mathrm{PN}$ description the spin precession equations were analyzed up to $0.5 \mathrm{PN}$ order. To the lowest order the angles between the total angular momentum and spin vectors are constant and spin-spin interaction causes additional harmonic dependence. The true anomaly parameterization proved to be useful in the description of eccentric orbits. In the circular and general cases linear and harmonic dependences of the angles describing the orientation of spins were found.

\subsection{Matched asymptotic expansion as a classical effective field theory by Kol}

In the talk it was explained how the method of classical effective field theory borrowed from quantum field theory by Goldberger and Rothstein [3] in the context of the motion of a compact object within a background whose typical length scale is much larger, is equivalent to matched asymptotic expansion, and moreover it offers additional insight [29]. Feynman diagrams, divergences, (dimensional) regularization, counter-terms and the Feynman gauge appeared. The ideas were demonstrated by the case of caged black holes (black holes within a compact dimension). Within this method the source is replaced by a 'black box' effective action. Another application of these ideas is to the inspiral problem of a binary system. The author presented a computation utilizing high energy physics methods of the radiation reaction force for the case of a scalar field.

\subsection{An analytic perturbation approach for classical spinning particle dynamics by Singh}

The author presented a perturbation method to analytically describe the dynamics of a classical spinning particle, based on the Mathisson-Papapetrou-Dixon equations of motion. By a power series expansion with respect to the particle's spin magnitude, it was demonstrated how to obtain an analytic representation of the particle's kinematic and dynamical degrees of freedom that is formally applicable to infinite order in the expansion parameter. Within this formalism, it is possible to identify a classical analog of radiative corrections to the particle's mass and spin due to spin-gravity interaction. The robustness of this approach was demonstrated by showing how to explicitly compute the first-order momentum and spin tensor components for arbitrary particle motion in a general spacetime background. Potentially interesting applications based on this perturbation approach were also considered. For more details see [30].

\subsection{Gravitational radiation from collapsing magnetized dust by Sotani, Yoshida and Kokkotas (reported by Sotani)}

The authors studied the influence of magnetic fields on the axial gravitational waves emitted during the collapse of a homogeneous dust sphere. It was found that while the energy emitted depends weakly on the initial matter perturbations it has strong dependence on the strength and the distribution of the magnetic field perturbations. The gravitational wave output of such 
a collapse can be up to an order of magnitude larger or smaller calling for detailed numerical 3D studies of collapsing magnetized configurations. More details are given in [31].

\subsection{Gravitational waveforms for compact binaries by Vasúth and Majár (reported by Vasúth)}

Among the promising sources of gravitational radiation are binary systems of compact stars. The detectable signal is characterized by different parameters of the system, e.g., rotation of the bodies and the eccentricity of the orbit. The authors presented a method to evaluate the gravitational wave polarization states for inspiralling compact binaries and considered eccentric orbits and the spin-orbit contribution in the case of two spinning objects up to 1.5 post-Newtonian order. In the circular orbit limit the presented results are in agreement with existing results. For more details see [32].

\subsection{On the Ricci tensor for non-stationary axisymmetric spacetimes by Kubeka}

The results on Ricci tensor for non-stationary axisymmetric spacetimes determined by Chandrasekhar [33] have been found to be incorrect both in the linear and in nonlinear regimes. However, the incorrectness of the Ricci tensor does not affect the well-known results on linear perturbations of a Schwarzschild black hole solution.

\section{Acknowledgments}

MV contribution was supported by OTKA grant no F049429. The work of SD, LP, IV and BW was supported by NSF grant no PHY-0555484.

\section{References}

[1] Buonanno A, Cook G B and Pretorius F 2007 Phys. Rev. D 75124018

[2] Boyle M, Brown D A, Kidder L E, Mroué A H, Pfeiffer H P, Scheel M A, Cook G B and Teukolsky S A 2007 Preprint gr-qc/0710.0158

[3] Goldberger W D and Rothstein I Z 2006 Phys. Rev. D 73104029

[4] Poisson E 2004 Living Rev. Rel. 76 http://www.livingreviews.org/lrr-2004-6

[5] Detweiler S 2005 Class. Quantum Grav. 22 S681

[6] Pound A and Poisson E 2008 Phys. Rev. D 77044012

[7] Pound A and Poisson E 2008 Phys. Rev. D 77044013

[8] Pound A, Poisson E and Nickel B G 2005 Phys. Rev. D 72124001

[9] Blaes O, Lee M H and Socrates A 2002 Astrophys. J. 578775

[10] Mino Y, Sasaki M and Tanaka T 1997 Phys. Rev. D 553457

[11] Quinn T C and Wald R M 1997 Phys. Rev. D 563381

[12] Detweiler S and Whiting B F 2003 Phys. Rev. D 67024025

[13] Keidl T S, Friedman J L and Wiseman A G 2007 Phys. Rev. D 75124009

[14] Ganz K, Hikida W, Nakano H, Sago N and Tanaka T 2007 Prog. Theor. Phys. 117 1041-60

[15] Barack L and Sago N 2007 Phys. Rev. D 75064021

[16] Dymnikova I G 1977 Astrophys. Space Sci. 51 229-34

[17] Dymnikova I G and Popov A K 1980 Astrophys. Space Sci. 68 487-94

[18] Schnittman J D and Bertschinger E 2004 Astrophys. J. 606 1098-111

[19] Whiting B F and Price L 2005 Class. Quantum Grav. 22 S589

[20] Tagoshi H, Ohashi A and Owen B 2001 Phys. Rev. D 63044006

[21] Kidder L, Will C and Wiseman A 1993 Phys. Rev. D 47 R4183

[22] Kidder L 1995 Phys. Rev. D 52821

[23] Faye G, Blanchet L and Buonanno A 2006 Phys. Rev. D 74104033 
[24] Blanchet L, Buonanno A and Faye G 2006 Phys. Rev. D 74104034

Blanchet L, Buonanno A and Faye G 2007 Phys. Rev. D 75049903 (erratum)

[25] Memmesheimer M, Gopakumar A and Schäfer G 2004 Phys. Rev. D 70104011

[26] Blanchet L and Schäfer G 1993 Class. Quantum Grav. 102699

[27] Arun K G, Blanchet L, Iger B R and Qusailah M S S 2008 Phys. Rev. D at press Preprint 0711.0250 Arun K G, Blanchet L, Iger B R and Qusailah M S S 2008 Phys. Rev. D at press Preprint 0711.0302

[28] Lämmerzahl C, Preuss O and Dittus H 2007 Is the physics in the Solar System really understood? Lasers, Clocks, and Drag-Free: Exploration of Relativistic Gravity in Space ed H Dittus, C Lämmerzahl and S G Turyshev (Berlin: Springer) p 75

[29] Kol B and Smolkin M 2008 Phys. Rev. D 77064033

[30] Singh D 2008 Gen. Rel. Grav. at press (Preprint 0706.0928v2)

[31] Sotani H, Yoshida S and Kokkotas K D 2007 Phys. Rev. D 75084015

[32] Vasúth M and Majár J 2007 Int. J. Mod. Phys. A 222405

[33] Chandrasekhar S 1975 Proc. R. Soc. A 343 289-98 